\title{
Hand-Held Force Magnifier for Surgical Instruments
}

\author{
George Stetten ${ }^{1,3,4}$, Bing Wu ${ }^{2}$, Roberta Klatzky ${ }^{2}$, John Galeotti ${ }^{3}$, Mel Siegel ${ }^{3}$, \\ Randy Lee ${ }^{4}$, Francis Mah ${ }^{5}$, Andrew Eller ${ }^{5}$, Joel Schuman ${ }^{5}$, and Ralph Hollis ${ }^{3}$ \\ ${ }^{1}$ Department of Bioengineering, University of Pittsburgh \\ ${ }^{2}$ Department of Psychology, Carnegie Mellon University \\ ${ }^{3}$ Robotics Institute, Carnegie Mellon University \\ ${ }^{4}$ Department of Biomedical Engineering, Carnegie Mellon University \\ ${ }^{5}$ Department of Ophthalmology, University of Pittsburgh School of Medicine \\ http: //www.vialab.org
}

\begin{abstract}
We present a novel and relatively simple method for magnifying forces perceived by an operator using a tool. A sensor measures the force between the tip of a tool and its handle held by the operator's fingers. These measurements are used to create a proportionally greater force between the handle and a brace attached to the operator's hand, providing an enhanced perception of forces between the tip of the tool and a target. We have designed and tested a prototype that is completely hand-held and thus can be easily manipulated to a wide variety of locations and orientations. Preliminary psychophysical evaluation demonstrates that the device improves the ability to detect and differentiate between small forces at the tip of the tool. Magnifying forces in this manner may provide an improved ability to perform delicate surgical procedures, while preserving the flexibility of a hand-held instrument.
\end{abstract}

Keywords: haptics, touch, robotic surgery, microsurgery, force magnifier, force-reflecting, steady hand.

\section{Introduction}

A need exists for improvement in the perception of forces by the sense of touch when using tools to perform delicate procedures. This is especially crucial in microsurgery. For example, surgeons routinely repair tiny blood vessels under a microscope that are far too delicate to be felt by the hand of the surgeon. Another key area for potential applications is ophthalmological surgery, in which we have recently been exploring techniques for image-guided intervention using optical coherence tomography [1]. Providing a useful sense of touch for such applications would improve outcome and increase safety.

Purely telerobotic systems such as the da Vinci $^{\circledR}$ Surgical System (Intuitive Surgical, Inc., Sunnyvale, CA) can provide motion-scaling, so that fine motion of the tool can be controlled by coarser motion of the operator's hand on the controls. Although force at the tool tip cannot be sensed by the operator in the current commercial da Vinci $^{\circledR}$ device, experimental systems have been tested that translate these forces into visual cues [2] as well as into vibrotactile feedback to the operators fingers [3]. 
A different, non-"tele"surgical approach has been demonstrated in several experimental systems, including the Force-Reflecting Motion-Scaling System created by Salcudean, et al. [4] [5], and the Steady Hand Robot described by Taylor, et al. [6][7]. These generate a magnified sense of touch by using a robotic arm that holds the surgical tool simultaneously with the surgeon, pushing and pulling as appropriate, to amplify the forces detected by small sensors between the handle of the tool and its tip. Because every force needs an opposing force, the robotic arm must be mounted somewhere, and being fairly massive, its weight must be supported by that mounting. Thus the magnified forces are created in these systems between the tool handle and subsequently the floor. To permit free motion of the tool by the surgeon, an elaborate remote-center-of-motion articulated robot arm is required, along with a control system to keep the tool moving naturally, as if controlled just by the operator, so that the surgeon can have something approaching the degrees of freedom and ease of manipulation that he/she is accustomed to with a freely held tool. Such systems are typically fairly extensive and complex. Issues involving the limited and congested workspace common in microsurgery raise serious challenges to practical deployment.

The desire to free robotic surgery devices from the floor-standing robotic arm has led to hand-held systems such as the Micron microsurgical instrument from Riviere's group, which uses piezoelectric actuators to move the tip relative to the handle, based on optical tracking of both the tip and handle [8]. The primary goal of Micron is to reduce the effects of hand tremor; it is not suited to provide a magnified sense of touch.

When the goal is to create additional forces for the operator to feel, some external frame to "push against" has generally been required. The field of haptic simulation faces the same dilemma of generating forces for the fingers to feel without anchoring the renderer to some solid base. Recent examples of more portable solutions include the "active thimble" described by Solazzi, et al. [9]. The device is entirely mounted on one hand. It attaches to the proximal part of the finger and reaches over to contact the fingertip, thus generating forces between two parts of the operator's own anatomy. As they describe it, "[a] limit of traditional kinesthetic interfaces is the difficulty to achieve a large workspace without a detriment of dynamic performance and transparency or without increasing the mechanical complexity. A possible solution to overcome this problem is to develop portable ungrounded devices that can display forces to the user hands or fingers."

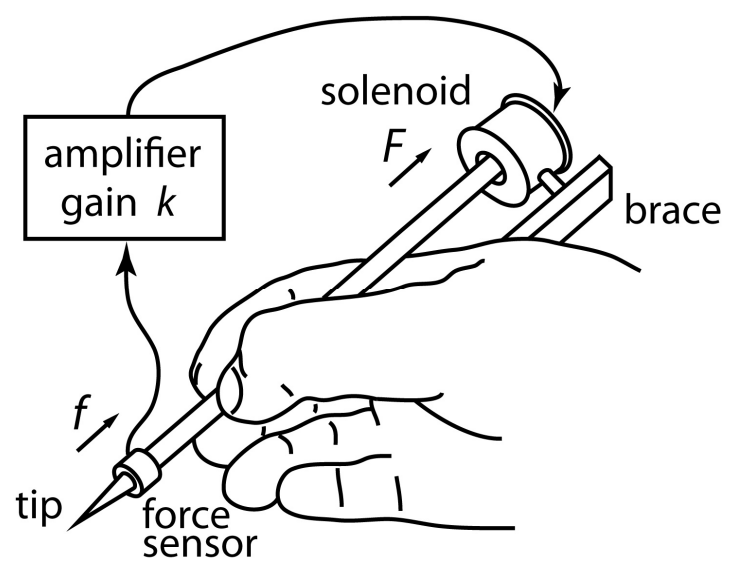

Fig. 1. The Hand-Held Force Magnifier (HHFM) uses a sensor to measure force $f$ between the handle and the tip, which is amplified to produce a force $F=k f$ in the same direction on the handle using a solenoid mounted on the back of the hand. 
In this paper we extend the concept of ungrounded haptic devices from purely virtual environments to real tools, by including a force sensor for interaction with an actual target. As in the Force-Reflecting and Steady Hand systems described above, we provide a magnified perception via the tool handle of forces sensed at the tool tip. Our device, however, does not require any freestanding apparatus, and instead produces forces between portions of the operator's own anatomy. The concept is shown in Fig. 1. A hand-held tool contains a sensor, which measures force $f$ between the handle and the tip. This signal is amplified to produce a force $F$ in the same direction on the handle of the tool using a solenoid mounted on the back of the hand. The human is, in effect, providing the moving platform from which the magnified forces are generated.

\section{Design and Operation of the Device}

Our Model-1 prototype of the Hand-Held Force Magnifier (HHFM) is illustrated in Fig. 2. In this first version, the tool tip is the small button on a force sensor (Honeywell FS01, 0-6.7 N). The tool handle is the body of a syringe attached to a piece of $1 / 4$ inch brass tubing containing a stack of 8 permanent rare-earth magnets (3/16" Radio Shack 64-1895) inserted into a custom solenoid (250 ft of 30 gauge wire, 25 ohms, approx. 2360 turns). Magnets are used rather than simply a ferromagnetic plunger, so that forces can be generated in both directions. The solenoid is attached by a dual gimbal to a brace, which is mounted to the back of a wrist splint strapped to the operator's right hand. The dual gimbal decreases friction within the solenoid and increases flexibility of use by permitting free rotation in azimuth and altitude, while maintaining a tight connection for force in the range direction. A control system (not shown) includes a push-pull linear amplifier capable of supplying $32 \mathrm{~V}$ at $2 \mathrm{~A}$, more than enough current to operate the solenoid over that voltage range. It employs simple proportional gain and permits adjustment of the offset to zero, to produce a force $F$ from the solenoid of up to $1 \mathrm{~N}$, proportional to, and in the same direction as, the force $f$ sensed at the tool tip (Figs. 1 and 2). Thus we can express the system's behavior as simply

$$
F=k f \text {. }
$$

The proportionality factor $k$ is adjustable from 0 to 5.8 in the Model-1. Above this level, the system becomes unstable, oscillating at approximately $400 \mathrm{~Hz}$. Particular values of $k$ (such as $k=2.4$ used in the experiments described below) can be determined by linearly fitting known forces imposed at the sensor to corresponding generated forces measured at the solenoid (see Fig. 5). The value of $k$ assumes that the plunger of the solenoid is in a fully extended state, which the operator is instructed to accomplish during normal operation by lightly pushing the tool handle forward before contacting a target. Higher values of $k$ result if the plunger is pulled within the solenoid, as this results in stronger attraction between the coil and the permanent magnets for a given current.

The Model-1 prototype in Fig. 2 is shown being used to push a spring from the side to bend it. With the gain $k$ set to 0 , the spring is felt through the tool to be subjectively quite easy to bend. With $k$ increased to maximum, the spring feels much harder to 


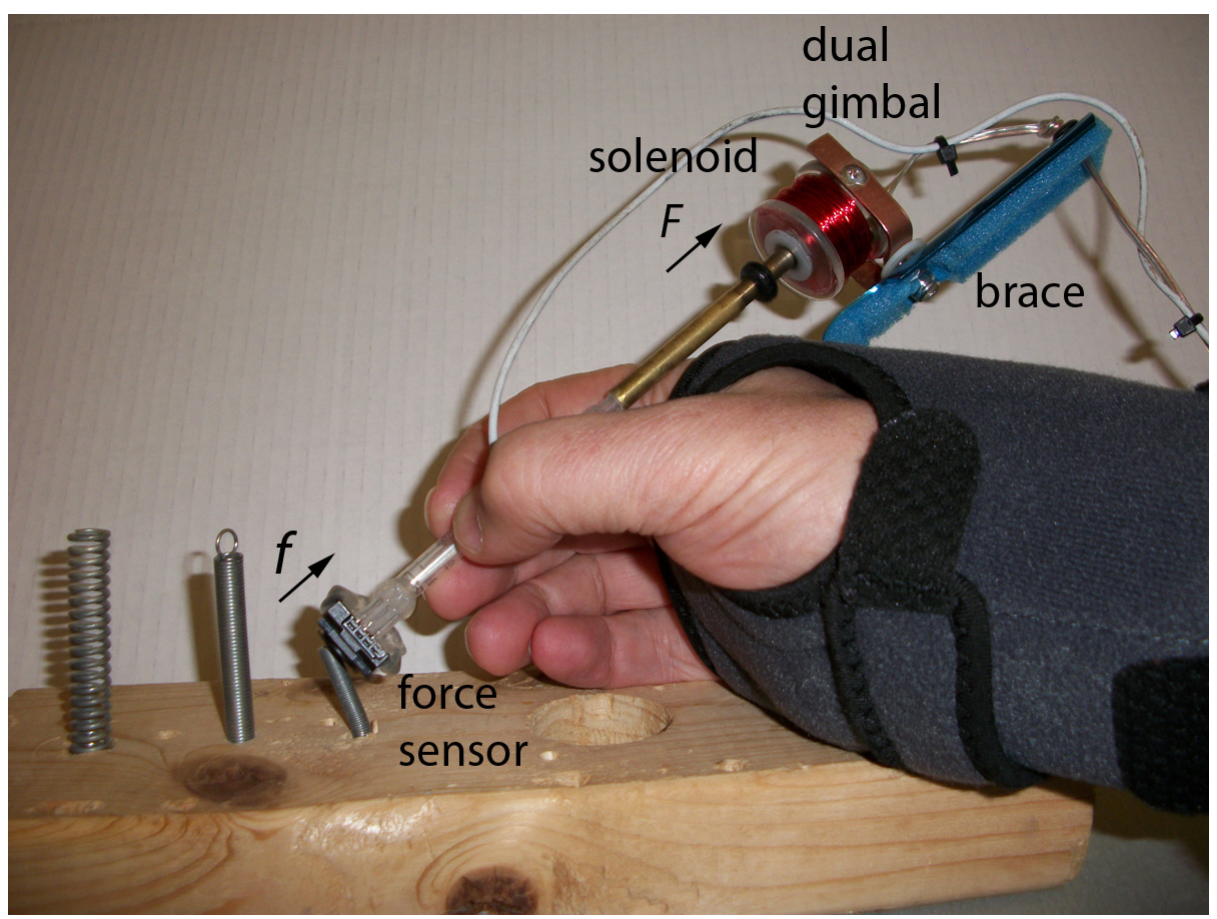

Fig. 2. Model-1 prototype of the Hand-Held Force Magnifier (HHFM). Here the operator "feels" a magnified version of the force generated by bending a spring.

bend. This haptic illusion is due to the fact that the fingertips must match not only the force of the spring $f$ but also of that of the solenoid $F$. Thus the device magnifies the operator's sensation of touch. We hypothesize that the operator can sense forces at the tool tip that are smaller than would otherwise be perceivable, and can control these smaller forces with greater delicacy by interacting with the magnified forces. We report on preliminary tests of this hypothesis in the following section.

\section{Psychophysics Experiments}

Two experiments were conducted to characterize the Hand-Held Force Multiplier (HHFM). In the first experiment, the participants' ability to sense the presence of a force (i.e. the force detection threshold) was measured with and without the use of the HHFM. In the second experiment, we used a method of magnitude estimation to characterize the impact of the device on the subjective force intensity. Both studies produced a measure of the perceptual magnification of force implemented by the HHFM.

Experimental setup: The experimental setup (Fig. 3) consisted of a magnetic levitation haptic device (MLHD) from Butterfly Haptics (Maglev 200TM, see 
http://butterflyhaptics.com) for rendering forces, a client computer for controlling stimuli and acquiring data, and a keypad for the participant to input responses.

The MLHD uses Lorentz forces for actuation, which arise from the electromagnetic interaction between current-carrying coils and magnets [10]. Since there are no motors, gears, bearings, or linkages present, it is free of static friction and able to generate forces precisely with a resolution of $0.02 \mathrm{~N}$. In the present study, the MLHD was connected to a client PC through a $100 \mathrm{Mbps}$ Ethernet cable. A multi-threaded application was run to command the MLHD to produce the desired stimuli and track the participant's responses from the keypad. Here the experimental stimuli were upward (counter-gravitational) forces ranging from 0.0-1.5 N. The forces were applied to the subject's hand while he or she held the HHFM (Fig. 3a) or an unmodified syringe (control condition) of the same diameter (Fig. 3b) in contact with the handle of the MLHD. The updating rate for force rendering was $2 \mathrm{kHz}$.

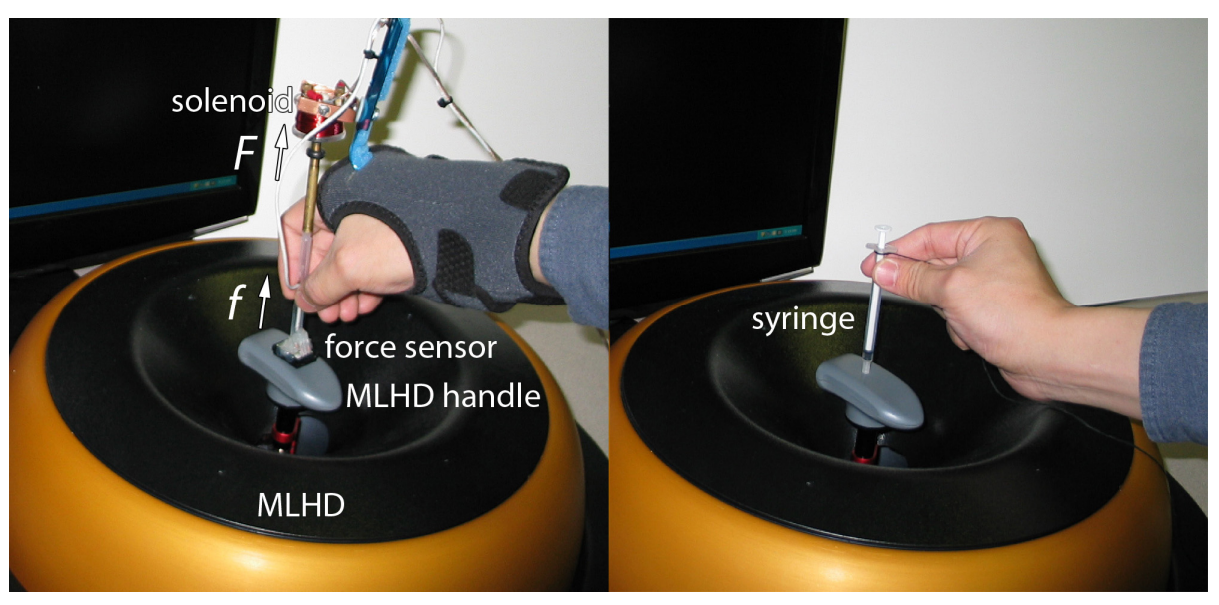

Fig. 3. Experimental setup for testing the Hand-Held Force Magnifier (HHFM). The user's perception of force was compared across the HHFM-on/off conditions (a) and control condition (b). A Magnetic Levitation Haptic Device (MLHD) was used to produce the experimental stimuli.

Experimental design: Participants were tested in three conditions in both experiments. In the HHFM-on and HHFM-off conditions, the participant put the device in his or her right hand and held the tubular portion between the thumb and index finger, as shown in Fig. 3a. He or she was instructed to hold the HHFM vertically, use it to press the MLHD handle, and so feel the vertical resisting force. Trials with the HHFM on or off were intermixed, and the experimenter controlled the state of the device with a switch as signaled by the computer without informing the participant. In the control condition (Fig. 3b), the participant held an unmodified syringe in the same way as the HHFM was held in the experimental conditions and felt the stimulus force transmitted through it. 
Experiment 1: Force detection with the HHFM. Six participants, four males and two females, aged between 22 and 35, participated in this experiment with informed consent. All were right-handed by selfreport and naïve to the purposes of this study.

Participants were tested individually with eyes closed. Each sat in a chair in front of the MLHD and adjusted the chair height to allow his or her right forearm to rest on the MLHD's top rim in a comfortable position. Active noise- cancelling headphones were worn to mask the sound from the environment. The participant was asked to hold the HHFM or the syringe and use it to touch the MLHD handle. A series of forces was then presented. In descending or ascending series, respectively, the initial force was clearly above $(0.3-0.4 \mathrm{~N})$ or below $(0.0 \mathrm{~N})$ the

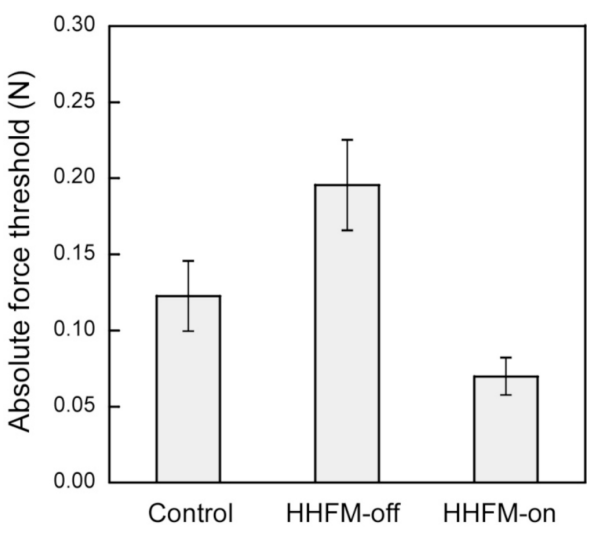

Fig. 4. Measured force detection thresholds. Error bars represent the standard error of the mean threshold, and thereafter the participant adjusted the force by pressing the "-" or "+" button until he or she felt that the stimulus had just disappeared or appeared. This procedure was repeated twice for each series. The threshold was then calculated as the mean value of the four threshold values.

Participants were tested first in the control condition and then in the HHFM on/off conditions. The testing order of HHFM-on and HHFM-off conditions was counterbalanced across participants.

Figure 4 plots the mean detection thresholds as a function of the experimental conditions. The mean threshold across all participants was $0.07 \mathrm{~N}$ in the HHFM-on condition, significantly lower than the threshold of $0.19 \mathrm{~N}$ when the HHFM was off $(\mathrm{t}(5)=3.99$, $\mathrm{p}=0.005$, one tailed paired $\mathrm{t}$-test). The threshold of $0.12 \mathrm{~N}$ obtained in the control condition was significantly less than the HHFM-off condition, $(\mathrm{t}(5)=5.85, \mathrm{p}=0.001)$, possibly because of the additional weight of the device. Thus comparisons between the HHFM-on and control may be contaminated by weight, but a marginal advantage for HHFM-on relative to control was still found, $(\mathrm{t}(5)=2.01$, $\mathrm{p}=0.05)$.

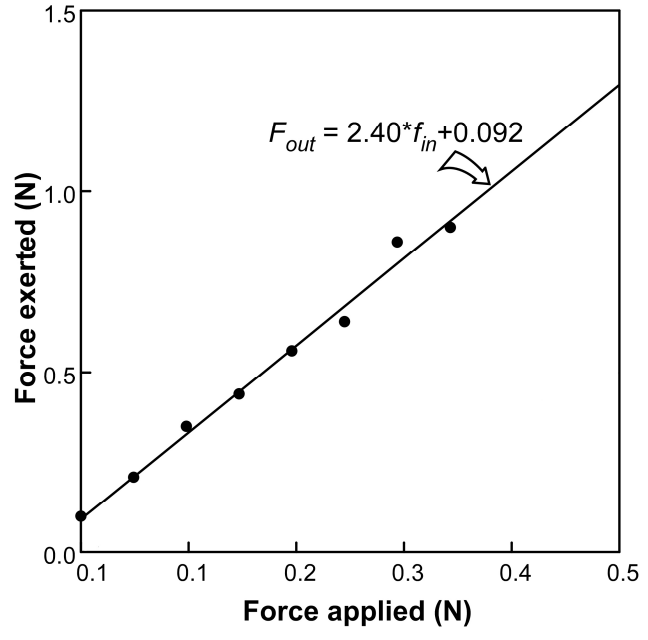

Fig. 5. Measured magnification factor $k$ of the HHFM represented as the slope of a linear fit to a series of force measurements at the sensor compared to those at the solenoid 
To quantify the perceptual magnification of the device, a measure was calculated as the ratio between the thresholds observed in the HHFM-on and HHFM-off conditions. Individual participants' perceived magnification yielded a confidence interval of $3.1 \pm 1.2$. This range includes the HHFMs actual total magnification, which we define to be $k+1$, or 3.4 , since the total force felt at the handle is $F+f$ (namely, the force from the solenoid plus that from the tip) and the value of $k$ was determined to be 2.4 (see Fig. 5).

Experiment 2: Force estimation with the HHFM. The same six participants took part in this experiment. The experimental setup was the same as previously. A magnitude estimation procedure [11] was used, by which the participants freely assigned numbers (integers or fractions) to force stimuli, in relation to their perceived intensity. Each force was to be judged in isolation, with the only restriction being that greater subjective intensity should produce a greater numerical value.

The force stimuli were $0.1,0.2,0.3, \& 0.4 \mathrm{~N}$. In addition, the forces of $0.5,1.0, \&$ $1.5 \mathrm{~N}$ were used in the control trials to produce a similar response range for both control and HHFM trials and thus reduce possible range effects resulting from the HHFM magnification. Each force was tested three times in each condition. The three repetitions occurred in different blocks in random order, with a 5-minute break between blocks. As before, the HHFM-on and HHFM-off trials were intermixed so that the participants had no knowledge of the status of the HHFM. The order of control and HHFM conditions was counter-balanced across subjects. The entire experiment lasted approximately one hour.
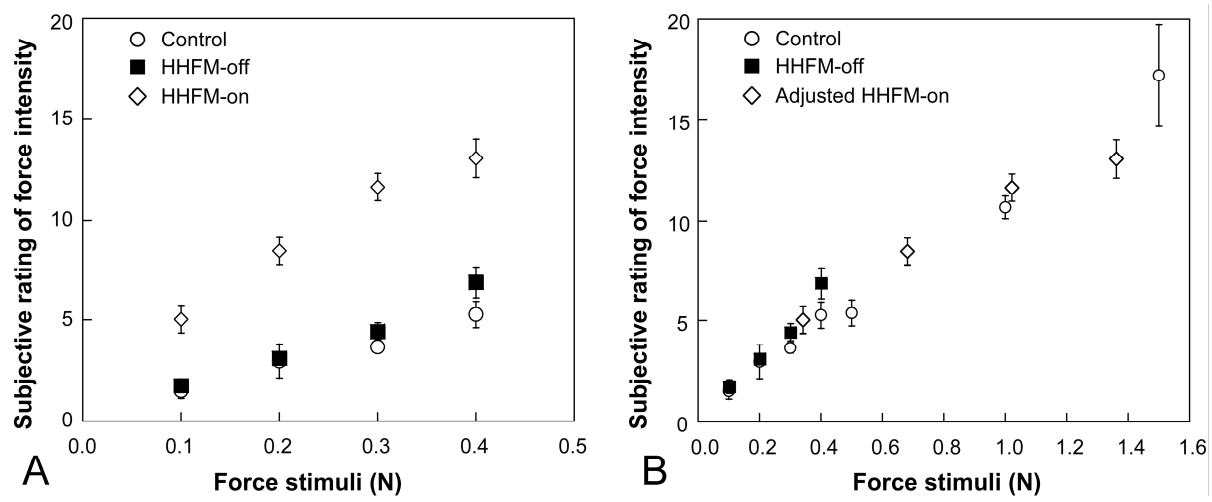

Fig. 6. (A) Mean judged magnitude for the stimulus forces. Error bars represent the standard error of the mean. (B) Plot of all data points, including the forces tested only in the control trials. Here the HHFM-on data were re-plotted versus the predicted output of the HHFM.

The data were normalized by dividing each judgment by the participant's mean within a given condition, and then multiplying by the grand mean for all participants. The three estimates from repeated measurements were averaged and taken as the estimated magnitude for each stimulus in each condition. Fig 6A shows the mean estimated magnitude, averaged across six subjects, for the forces tested in the three conditions. Perceptual magnification was then estimated for each participant as the 
slope of a linear regression relating the mean HHFM-on magnitude to the HHFM-off magnitude for each stimulus. The participants' perceived magnification ranged from 1.85 to 2.40 with a mean of $2.14 \pm 0.18$, somewhat less than the HHFM's total magnification factor determined above to be 3.4. If the HHFM-on curve was rescaled by multiplying each objective stimulus value ( $\mathrm{x}$-axis point) by the total magnification factor, as shown in Fig 6B, it essentially coincided with the curve obtained in the control condition.

Three two-way (force $\times$ device) repeated-measure ANOVAs were performed on the raw data to assess the effects of the HHFM on perceived force magnitude. The comparison of the HHFM-off and control data found only a significant main effect for force $(F(3,15)=16.61, p<0.001)$, but showed no device effects $(F(1,5)=0.18, p=0.69)$ or significant interaction $(\mathrm{F}(3,15)=0.67, \mathrm{p}=0.59)$. That is, the additional weight of the HHFM did not affect subjective magnitude in this supra-threshold task, as it did the threshold in the previous experiment. The same ANOVA comparing HHFM-on to control showed, in contrast, higher magnitudes for the HHFM-on $(F(1,5)=20.76$, $\mathrm{p}=0.006)$ and a significant force $\times$ device interaction $(\mathrm{F}(3,15)=4.45, \mathrm{p}=0.02)$ as well as a significant main effect for force $(\mathrm{F}(3,15)=18.40, \mathrm{p}<0.001)$. Similarly, the ANOVA comparing HHFM-on and HHFM-off showed greater magnitudes for the HHFM-on condition $(\mathrm{F}(1,5)=37.31, \mathrm{p}=0.002)$, a significant interaction $(\mathrm{F}(3,15)=4.54$, $\mathrm{p}=0.02)$ and a significant main effect for force $(\mathrm{F}(3,15)=15.11, \mathrm{p}<0.001)$.

\section{Discussion}

Our psychophysical experiments clearly show that percepts of force at both threshold and supra-threshold levels are rescaled when using the HHFM, demonstrating that the force magnification induced by the device is well perceived by human users. The perceptual force magnifications observed in the two experiments are less than the actual magnification, particularly in the force-estimation experiment. This loss of force magnification through perceptual transmission might be due to the friction observed in the custom solenoid. Two additional issues should also be noted in interpreting this result. First, the perceptual magnification was estimated here by a linear regression analysis, whereas the curves in Fig 6a were best fitted by power functions with exponents of 0.71 and 0.96 , respectively, for the HHFM-on and HHFM-off data. Without considering such compressive nonlinearity in force perception, the magnification derived from the linear fitting of HHFM-on vs. HHFM-off data would be underestimated. Second, since the HHFM is pinch-held by the user as illustrated in Figs. 2 and 3, force perception is significantly influenced by the surface texture at the contact locations [12]. Essentially, the smoother the surface texture, the greater the grip force required and the more difficult it is to perceive the axial force. In the current prototype, the handle of the HHFM is the body of a syringe that has fine surface texture. This may contribute to the reduced perception of axial forces transmitted along the HHFM handle. In future research we will examine these issues further and revise the HHFM design accordingly to maximize the operator's perceptual magnification.

The following anecdotal observation surprised us. When pushing against the spring (see Fig. 2), we had expected the optimal motion to be that of just the fingertips moving the tool relative to the solenoid. Instead, we found that the most convincing 
sensation of magnified stiffness in the spring is achieved by moving the entire device, including the solenoid, with the muscles of the upper arm and shoulder. This makes little sense from a Newtonian point of view, since the device is only capable of generating forces between the solenoid and the fingertips. It indicates that force perception at the fingertips is influenced by sensorimotor integration between the fingers, hands, arms, and shoulders.

It is too early to predict exactly which clinical applications may prove most suitable for the HHFM, or to know what types of tool-tips will be most useful. At present, we are primarily considering applications in ophthalmological surgery. This is, in part, due to the authors' ongoing collaboration with ophthalmological surgeons in developing techniques for image guidance for eye surgery, but also because eye surgery is so clearly a realm in which many very delicate operations are performed. Two primary categories of eye surgery are procedures on the cornea and those on the retina. Corneal procedures are generally less delicate than retinal procedures, and are closer to the surface and therefore more accessible, but both corneal and retinal procedures are performed under the surgical microscope, involve extremely fine motor skills, and are often devoid of a useful sense of touch.

Corneal Surgery: Several corneal procedures are used to treat glaucoma, including trabeculotomy and canaloplasty, which create communication between the anterior chamber and Schlemm's canal, decreasing resistance to outflow. Trabeculotomy involves using a tiny blunt device to tear through the inner wall of Schlemm's canal and surrounding trabecular meshwork. Canaloplasty involves injecting viscoelastic material through a microcatheter along the entire $360^{\circ}$ path of the canal and placing a stent suture. A third, experimental technique involves insertion of permanent stents into the canal $a b$ interno, bypassing the trabecular meshwork to permit aqueous humor direct access to Schlemm's canal. These manipulations could be aided by an enhanced sense of touch provided by HHFM-based tools. Another candidate is keratectomy to repair corneal dystrophies, scars, or damage from foreign bodies, as well as Deep Anterior Lamellar Keratectomy to treat the gradual bulging of the cornea into a conical shape. In this latter procedure, perforation of Descemet's membrane often leads to mandatory conversion to Penetrating Keratoplasty a more radical procedure. It may be that greater sensitivity to forces could prevent this. Finally, corneal transplant commonly involves a Descemet's Stripping Automated Endothelial Keratoplasty (DSAEK), which maintains the patient's own outer cornea, replacing just the inner portion with donor tissue inserted through a small slit. The procedure is extremely delicate, both in the actual transplantation and in the prior harvesting of the graft from the donor.

Retinal Surgery: Procedures on the retina are generally even more delicate than those in the cornea, and have the added constraint that the tool must first be inserted through the sclera to reach the retina. The particular procedures we are considering involve peeling one of several types of membranes off the retina. An epiretinal membrane (ERM) is a glial cell proliferation that can cause blurred or distorted central vision. After removing the vitreous, the surgeon scores the ERM with a barb and then pulls it up with forceps or a diamond-dusted scraper. Another type of membrane, the internal limiting membrane (ILM), is a naturally occurring extracellular matrix that guides axons during the formation of the retina but is not needed thereafter. It has 
been shown that removing the (ILM) increases the closure rate of holes in the macula (the highest resolution portion of the fovea) that can significantly impede vision. Observing a retinal surgeon remove such membranes from the retina with nothing but visual feedback is instructive as to what is possible freehand. In particular, extremely fine control of tiny motion is possible when stabilized by insertion of the hand-held tool through the sclera. Also critical is the position of the surgeon's hand resting on the patient. Clearly, forces generated within the hand are central to such fine motor control. In particular, the act of gently peeling a membrane away from the retina, preferably in one piece without tearing it, seems to be a good candidate for increased sensitivity to minute forces.

In the face of such extremely delicate procedures, the Model-1 prototype leaves much room for improvement. As mentioned above, magnifications only up to 5.8 are presently possible without instability. Higher magnification may be useful in microsurgery, and would likely be possible with the inclusion of differential and/or integral gain, using a classical Proportional-Integral-Differential (PID) controller. The present amplifier can send current either way through the solenoid, so that by using a bidirectional force sensor we will be able to test tools that pull, such as hooks, as well as push. It is theoretically possible to also magnify non-axial forces and even torques with the HHFM. The present location of the brace may make it difficult to efficiently produce such lateral forces at the tool tip. A certain amount of natural lateral force magnification is, in fact, already present at the fingertips, due to the relative lengths of the lever arms. However, it is clear that the alternate approach of using an external robotic arm (as described in the Introduction) would make such lateral forces and torques easier to generate. Other improvements to the HHFM, such as the production of multiple simultaneous forces, as between the tips of a forceps, are also candidates for inclusion in future prototypes.

It is likely that when extremely small forces are involved, the external robotic arm may be advantageous due to the added stability of a grounded platform. Conversely, patient movement relative to such a stationary platform may prove a serious problem, favoring smaller, truly telerobotic systems that brace against the patient to generate forces for micromanipulation, such as the HeartLander system for minimally invasive cardiac surgery [13]. In the spectrum of such devices, it may be that the HHFM is best suited for an intermediate range of forces, perhaps in the cornea or elsewhere in the body, where structures of the appropriate range of delicacy are targets for surgical procedures.

\section{Conclusion}

The major contribution of our work, we believe, is to provide a magnified sense of touch without requiring an external robotic arm. The force that was generated between the operator's hand and the floor by the robotic arm is replaced by a force generated between two locations on the operator's hand, freeing the design to permit a small, light, hand-held device. Such a device may be easier to manipulate and more practical to deploy in certain clinical settings, where fewer degrees of freedom and intermediate force sensitivity are acceptable trade-offs for greater flexibility and independence of operation. 
Acknowledgments. This work was funded by a NIH K99 grant 5K99EB008710 and a William Kepler Whiteford Professorship at the University of Pittsburgh.

\section{References}

1. Galeotti, J., Sajjad, A., Wang, B., Kagemann, L., Shukla, G., Siegel, M., Wu, B., Klatzky, R., Wollstein, G., Schuman, J., Stetten, G.: The OCT penlight: In-situ image guidance for microsurgery, SPIE Medical Imaging, paper \#7625-1 (2010)

2. Bethea, B., Okamura, A., Kitagawa, M., Fitton, T., Cattaneo, S., Gott, V., Baumgartner, W., Yuy, D.: Application of Haptic Feedback to Robotic Surgery. J. Laparoendosc Adv. Surg. Tech. A 14(3), 191-195 (2004)

3. Kuchenbecker, K.J., Gewirtz, J., McMahan, W., Standish, D., Martin, P., Bohren, J., Mendoza, P.J., Lee, D.I.: VerroTouch: High-frequency acceleration feedback for telerobotic surgery. In: Kappers, A.M.L., van Erp, J.B.F., Bergmann Tiest, W.M., van der Helm, F.C.T. (eds.) EuroHaptics 2010. LNCS, vol. 6191, pp. 189-196. Springer, Heidelberg (2010)

4. Salcudean, S.E., Yan, J.: Motion scaling teleoperating system with force feedback suitable for microsurgery, U.S. Patent 5,382,885 (1995)

5. Salcudean, S.E., Yan, J.: Towards a Force-Reflecting Motion-Scaling System for Microsurgery. In: IEEE International Conference on Robotics and Automation, San Diego, California (1994)

6. Taylor, R., Barnes, A., Kumar, R., Gupta, P., Wang, Z., Jensen, P., Whitcomb, L.: A steady-hand robotic system for microsurgical augmentation. In: Taylor, C., Colchester, A. (eds.) MICCAI 1999. LNCS, vol. 1679, pp. 1031-1041. Springer, Heidelberg (1999)

7. Fleming, I., Balicki, M., Koo, J., Iordachita, I.I., Mitchell, B., Handa, J., Hager, G.D., Taylor, R.: Cooperative robot assistant for retinal microsurgery. In: Metaxas, D., Axel, L., Fichtinger, G., Székely, G. (eds.) MICCAI 2008, Part II. LNCS, vol. 5242, pp. 543-550. Springer, Heidelberg (2008)

8. Tabars, J., MacLachlan, R., Ettensohn, C., Riviere, C.: Cell Micromanipulation with an Active Handheld Micromanipulator. In: 32nd Annual International Conference of the IEEE EMBS, Buenos Aires, Argentina (2010)

9. Solazzi, M., Frisoli, A., Bergamasco, M.: Design of a Novel Finger Haptic Interface for Contact and Orientation Display. In: IEEE Haptics Symposium, Waltham, Massachusetts (March 25-26, 2010)

10. Hollis, R.L., Salcudean, E.: Lorentz Levitation Technology: A New Approach to Fine Motion Robotics, Teleoperation, Haptic Interfaces, and Vibration Isolation. In: 5th International Symposium on Robotics Research, Hidden Valley, PA, October 1-4 (1993)

11. Stevens, S.S.: Psychophysics: Introduction to its Perceptual, Neural, and Social Prospects. John Wiley, New York (1975)

12. Flanagan, J.R., Wing, A.M.: Effects of surface texture and grip force on the discrimination of hand-held loads. Perception \& Psychophysics 59, 111-118 (1997)

13. Ota, T., Patronik, N., Riviere, C., Zenati, M.A.: Percutaneous Subxiphoid Access to the Epicardium Using a Miniature Crawling Robotic Device. Innovations 1(5), 227-231 (2006) 